\title{
Primeiro registro de Anthonomus tomentosus (Faust) (Coleoptera: Curculionidae) na Amazônia Oriental
}

\author{
Ricardo Adaime ${ }^{\circledR}$, Rildo Pessoa Almeida ${ }^{2}$, José Francisco Pereira1, \\ Alberto Luiz Marsaro Júnior ${ }^{3}$ \& Germano Henrique Rosado-Neto ${ }^{4}$
}

1. Embrapa Amapá - Laboratório de Proteção de Plantas - Macapá, AP, Brasil. 2. Faculdade de Macapá. 3. Embrapa Trigo - Passo Fundo - RS, Brasil. 4. Universidade Federal do Paraná - Curitiba- PR - Brasil.

\section{EntomoBrasilis 12 (2): 84-87 (2019)}

Resumo. Anthonomus tomentosus (Faust) (Coleoptera: Curculionidae) é registrado pela primeira vez na Amazônia Oriental, a partir de frutos de Malpighia emarginata DC. coletados no estado do Amapá, Brasil. Neste trabalho também são apresentados sinais de danos aos frutos e a distribuição geográfica de A. tomentosus no Brasil.

Palavras-chave: Acerola; Curculionoidea; Fruticultura; Praga quarentenária; Gorgulho.

\section{First record of Anthonomus tomentosus (Faust) (Coleoptera: Curculionidae) in the Eastern Amazon}

Abstract. Anthonomus tomentosus (Faust) (Coleoptera: Curculionidae) is recorded for the first time in the Eastern Amazon, from fruits of Malpighia emarginata DC. collected in the state of Amapá, Brazil. In this work we also present the signals damages on the fruits and the geographical distribution of $A$. tomentosus in Brazil.

Keywords: Acerola; Curculionoidea; Fruit production; Quarantine pest; Weevil.

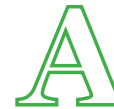
acerola (Malpighia emarginata DC., Malpighiaceae) tem despertado o interesse dos fruticultores devido ao seu alto teor natural de vitamina $\mathrm{C}$ e sua grande capacidade de aproveitamento industrial (NogUEIRA et al. 2002; FreITAS et al. 2006). Em decorrência disso, existem plantios comerciais em praticamente todos os Estados brasileiros (ALvES 1996), tornando o país o maior produtor, consumidor e exportador de acerola no mundo (CALGARo \& BRAGA 2012). Atualmente, a produção nacional de acerola é de 142.991,56 toneladas, com cultivos distribuídos em 6.645 propriedades rurais (IBGE 2017).

Foi detectada no Brasil, no estado de Roraima, uma praga que pode causar significativos prejuízos socioeconômicos à cultura da acerola, o coleóptero Anthonomus tomentosus (Faust) (Coleoptera: Curculionidae) (Figura 1a), obtido de frutos provenientes dos municípios de Boa Vista, Mucajaí, Pacaraima e Normandia (MARSARO JÚNIOR et al. 2017). Antes desse registro, a praga só havia sido relatada na Venezuela e Trinidad (CLARK 1987). O inseto era considerado praga quarentenária ausente no Brasil, de acordo com a Instrução Normativa $n^{\circ} 41 / 2008$ do Ministério da Agricultura, Pecuária e Abastecimento (BRASIL 2008). Porém, recentemente, passou a ser considerado praga quarentenária presente no país, com ocorrência exclusiva no estado de Roraima, por meio da Instrução Normativa $\mathrm{n}^{0}$ 38/2018 (BRASIL 2018).

Edited by:

Rodrigo Souza Santos

Article History:

Received: 21.ix.2018

Accepted: 05.iv.2019
As larvas de $A$. tomentosus causam injúrias nos frutos de $M$. emarginata, provocando perdas qualitativas e quantitativas ao se alimentarem do mesocarpo (Figura 1b). Os frutos infestados tornam-se deformados (Figura 1c), comprometendo seu valor como produto in natura (MARSARO JÚNIOR et al. 2017).

Com o intuito de verificar a possível presença de $A$. tomentosus no estado do Amapá, foram coletadas quinze amostras de frutos de $M$. emarginata, diretamente das plantas, em quintais residenciais nos municípios de Macapá, Santana e Mazagão, entre abril e maio de 2018 (Tabela 1). Os frutos foram transportados para o Laboratório de Proteção de Plantas da Embrapa Amapá, em Macapá, AP, onde foram contabilizados, pesados e acondicionados sobre areia esterilizada, em potes plásticos com tampas teladas com tecido voil, e mantidos em câmara climatizada $\left(26 \pm 1{ }^{\circ} \mathrm{C}, 60 \pm 10 \%\right.$ UR e fotofase de 12 h). A cada dez dias os potes eram inspecionados para verificar possíveis insetos emergidos.

Foram obtidos 151 besouros curculionídeos a partir de cinco amostras procedentes de Macapá e de uma oriunda de Santana (Tabela 1). Os insetos obtidos foram acondicionados em tubos contendo álcool 70\% e enviados ao Laboratório de Entomologia do Departamento de Zoologia da Universidade Federal do Paraná (UFPR), em Curitiba, Paraná, Brasil, para identificação taxonômica, aos cuidados do Prof. Dr. Germano Henrique
Corresponding author:

Ricardo Adaime

乃 ricardo.adaime@embrapa.br

(1) https://orcid.org/0000-0001-8044-3976
Funding agencies:

$\checkmark$ Without funding declared 

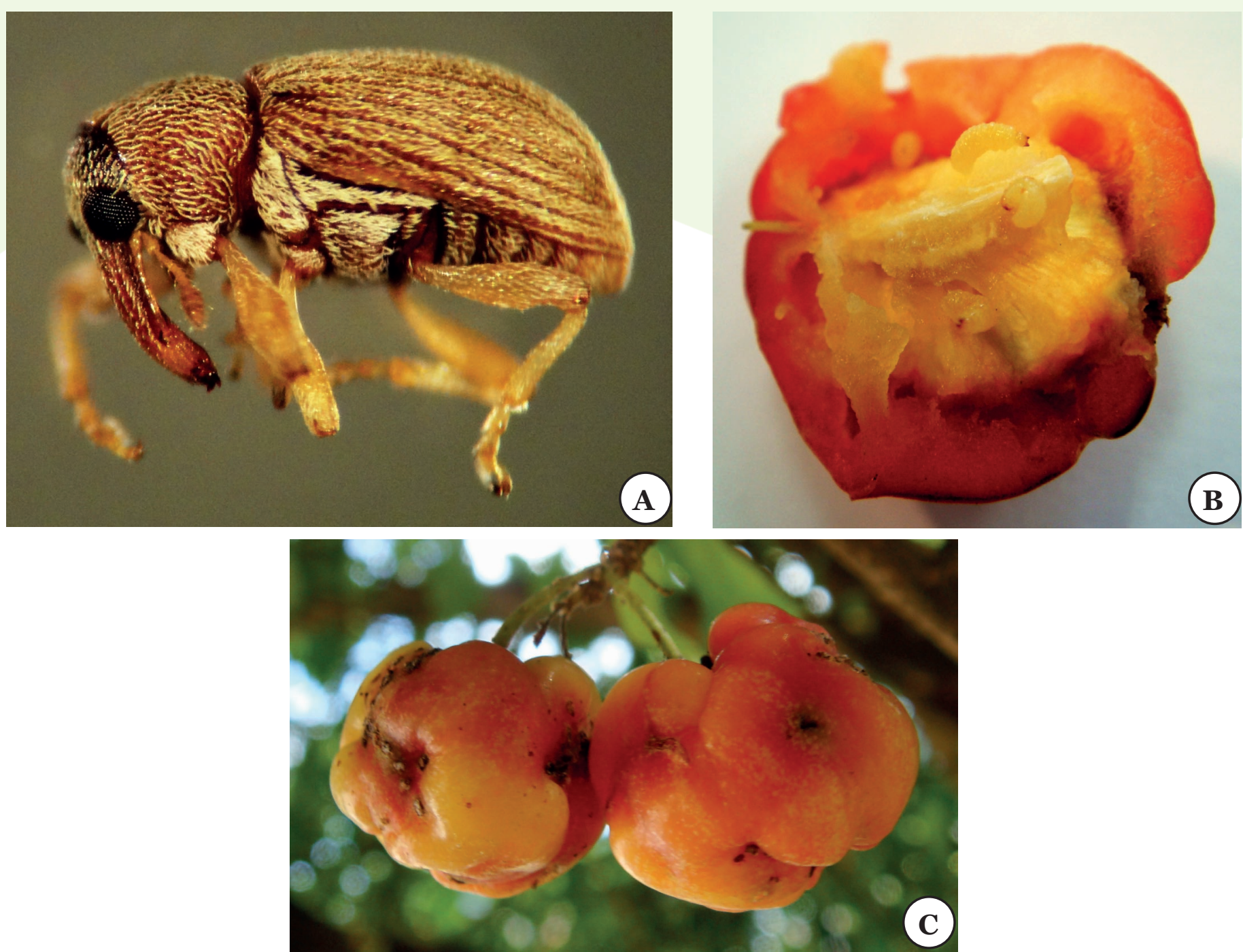

Figura 1. Anthonomus tomentosus. a) macho; b) injúrias no mesocarpo de fruto de acerola ocasionadas por larvas; c) injúrias no epicarpo de frutos de acerola ocasionadas por larvas. Fotos: Marsaro Júnior, AL.

Tabela 1. Amostras de frutos de Malpighia emarginata coletadas em municípios do estado do Amapá e espécimes de Anthonomus tomentosus obtidos.

\begin{tabular}{|c|c|c|c|c|c|c|c|}
\hline Amostras & $\begin{array}{l}\text { Data de } \\
\text { coleta }\end{array}$ & Municípios & Localidades & Coordenadas & $\begin{array}{c}\text { Peso de } \\
\text { frutos (g) }\end{array}$ & $\begin{array}{l}\text { Número } \\
\text { de frutos } \\
\text { (n) }\end{array}$ & $\begin{array}{l}\text { Insetos } \\
\text { obtidos } \\
\text { (n) }\end{array}$ \\
\hline 1 & $20 / 04 / 2018$ & Macapá & Bairro Novo Horizonte & $00^{\circ} 06^{\prime} 01^{\prime \prime} \mathrm{N}-51^{\circ} \mathrm{O} 2^{\prime} 4 \mathrm{O}^{\prime \prime} \mathrm{W}$ & $500^{\mathrm{a}}$ & $\mathrm{nc}^{\mathrm{b}}$ & 51 \\
\hline 2 & $20 / 04 / 2018$ & Macapá & Bairro Jardim Felicidade & $\mathrm{Oo}^{\circ} \mathrm{O} 4^{\prime} 44^{\prime \prime} \mathrm{N}-51^{\circ} \mathrm{O} 3^{\prime} 41^{\prime \prime} \mathrm{W}$ & $500^{\mathrm{a}}$ & $n c^{b}$ & 47 \\
\hline 3 & 09/05/2018 & Macapá & Bairro Jardim Felicidade & $\mathrm{Oo}^{\circ} \mathrm{O} 4^{\prime} 44^{\prime \prime} \mathrm{N}-51^{\circ} \mathrm{O} 3^{\prime} 41^{\prime \prime} \mathrm{W}$ & 215 & 51 & 50 \\
\hline 4 & 09/05/2018 & Macapá & Bairro Novo Horizonte & $\mathrm{oo}^{\circ} \mathrm{O} 5^{\prime} \mathrm{O} 6^{\prime \prime} \mathrm{N}-51^{\circ} \mathrm{O} 2^{\prime} 39^{\prime \prime} \mathrm{W}$ & 141 & 26 & 1 \\
\hline 5 & 09/05/2018 & Macapá & Bairro Novo Horizonte & 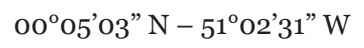 & 197 & 50 & o \\
\hline 6 & 09/05/2018 & Macapá & Bairro Novo Horizonte & $00^{\circ} \mathrm{O} 6^{\prime} 03 " \mathrm{~N}-51^{\circ} \mathrm{O} 2^{\prime} 4 \mathrm{O}^{\prime \prime} \mathrm{W}$ & 121 & 35 & o \\
\hline 7 & 09/05/2018 & Macapá & Abacate da Pedreira & $\mathrm{oo}^{\circ} 16^{\prime} 32^{\prime \prime} \mathrm{N}-5 \mathrm{O}^{\circ} 55^{\prime} 36^{\prime \prime} \mathrm{W}$ & 251 & 53 & o \\
\hline 8 & 09/05/2018 & Macapá & Abacate da Pedreira & $\mathrm{oo}^{\circ} 16^{\prime} 26^{\prime \prime} \mathrm{N}-5 \mathrm{O}^{\circ} 55^{\prime} 21^{\prime \prime} \mathrm{W}$ & 68 & 23 & o \\
\hline 9 & 09/05/2018 & Macapá & Abacate da Pedreira & $\mathrm{Oo}^{\circ} 16^{\prime} 26^{\prime \prime} \mathrm{N}-5 \mathrm{O}^{\circ} 55^{\prime} 21^{\prime \prime} \mathrm{W}$ & 194 & 50 & 1 \\
\hline 10 & 09/05/2018 & Macapá & Abacate da Pedreira & $\mathrm{oo}^{\circ} 14^{\prime} 38^{\prime \prime} \mathrm{N}-50^{\circ} 57^{\prime} 43^{\prime \prime} \mathrm{W}$ & 260 & 50 & o \\
\hline 11 & 09/05/2018 & Santana & - & $\mathrm{OO}^{\circ} \mathrm{O} 1^{\prime} 41^{\prime \prime} \mathrm{S}-51^{\circ} 10 \mathrm{O} 2 ” \mathrm{~W}$ & 257 & 50 & o \\
\hline 12 & 09/05/2018 & Santana & - & $\mathrm{OO}^{\circ} \mathrm{O} 1^{\prime} 41^{\prime \prime} \mathrm{S}-51^{\circ} 10 \mathrm{O} 2 ” \mathrm{~W}$ & 192 & 50 & o \\
\hline 13 & 09/05/2018 & Santana & - & $00^{\circ} 01^{\prime} 05 " \mathrm{~S}-51^{\circ} 10^{\prime} 12 ” \mathrm{~W}$ & 58 & 24 & 1 \\
\hline 14 & $16 / 05 / 2018$ & Mazagão & Camaipi & $\mathrm{oo}^{\circ} \mathrm{O} 8^{\prime} 15^{\prime \prime} \mathrm{S}-51^{\circ} 21^{\prime} \mathrm{Oo} " \mathrm{~W}$ & 170 & 37 & o \\
\hline 15 & $16 / 05 / 2018$ & Mazagão & Rodovia Mazagão & $\mathrm{Oo}^{\circ} 08^{\prime} 45^{\prime \prime} \mathrm{S}-51^{\circ} 22^{\prime} 11^{\prime \prime} \mathrm{W}$ & 165 & 37 & o \\
\hline
\end{tabular}

aPeso aproximado

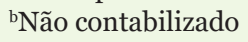


Rosado-Neto. No laboratório, os insetos foram montados em alfinetes entomológicos e examinados sob microscópio estereoscópico. Após comparação com exemplares coletados no estado de Roraima e estudo dos caracteres morfológicos de acordo com a chave taxonômica publicada por CLARK (1987), concluiu-se que as amostras se tratavam de A. tomentosus. Os espécimes foram depositados na Coleção de Entomologia do Departamento de Zoologia, Setor de Ciências Biológicas da Universidade Federal do Paraná.

Este é o primeiro registro de A. tomentosus na Amazônia Oriental e o segundo registro no Brasil, o que indica que a praga está ampliando sua distribuição na região (Figura 2). Por outro lado, é possível que a praga já estivesse presente, sem ter sido detectada. Considerando o potencial da praga para causar prejuízos socioeconômicos à cultura da acerola, são necessários levantamentos, principalmente nos Estados que fazem divisa com Amapá e Roraima, bem como naqueles que concentram a maior produção de acerola no país. Adicionalmente, é imperioso envidar esforços no sentido de desenvolver estratégias de controle da praga, como por exemplo o controle biológico das larvas, que pode ser realizado por meio de parasitoides
(Hymenoptera) das famílias Braconidae, Pteromalidae e Eupelmidae, conforme relatado por JARQUín-LóPEZ et al. (2011) para Anthonomus sisyphus Clark; e/ou investigar possíveis feromônios de agregação em adultos, uma vez que esses compostos já foram encontrados em diversas outras espécies de Anthonomus (Ambrogi et al. 2009), que poderiam ser sintetizados e incorporados em armadilhas de captura.

Analisando de uma maneira ampla, a região amazônica é extremamente vulnerável à introdução de pragas presentes nos países e territórios de fronteira (LEMos et al. 2011). A região tem sido a porta de entrada para diversas pragas quarentenárias de alto potencial de impacto socioeconômico e ambiental que, se disseminadas para áreas de produção, podem ocasionar sérias perdas ao agronegócio nacional, não apenas pela redução na produção, como também pela imposição de barreiras fitossanitárias para as exportações brasileiras e aumento do custo da produção devido aos métodos de controle utilizados (MORAIS et al. 2016). Isso reforça a necessidade de maiores investimentos em defesa fitossanitária no Brasil, especialmente nas regiões de fronteira (MARSARO JÚNIOR et al. 2017).

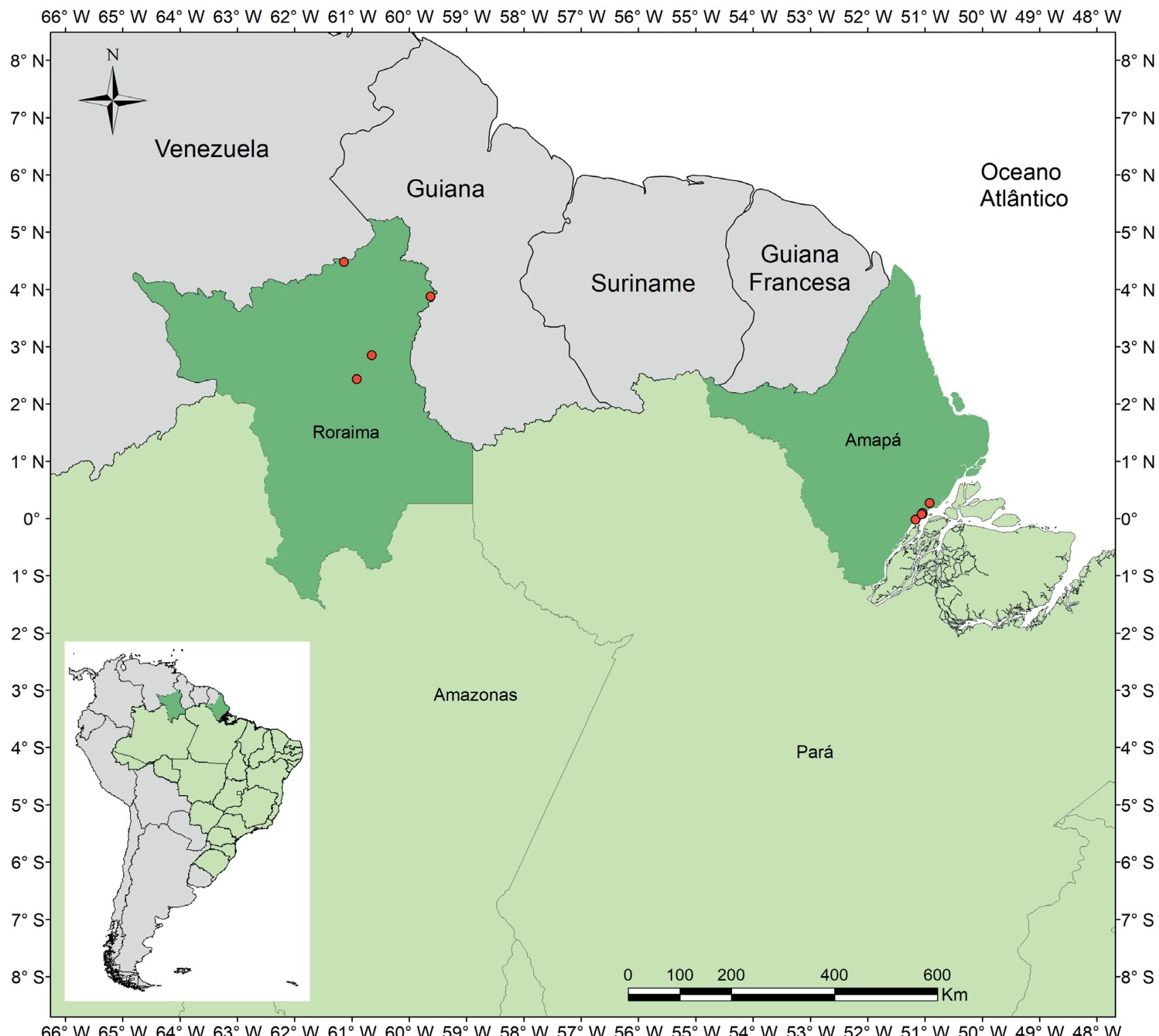

Figura 2. Distribuição geográfica de Anthonomus tomentosus no Brasil.

\section{AGRADECIMENTOS}

Os autores são gratos à doutoranda Maria do Socorro Miranda de Sousa (Programa de Pós-graduação em Biodiversidade Tropical,
Universidade Federal do Amapá), pela contribuição aos originais deste manuscrito. 


\section{REFERÊNCIAS}

Alves, RE, 1996. Características das frutas para exportação, pp. 9-12. In: Gorgatti Netto, A., E.F.G. Ardito \& E.E. Garcia (Eds.). Acerola para exportação: procedimentos de colheita e pós-colheita. Brasília: EMBRAPA-SPI, 30 p.

Ambrogi, BG, DM Vidal, PHG Zarbin \& GH Rosado-Neto, 2009. Feromônios de agregação em Curculionidae (Insecta: Coleoptera) e sua implicação taxonômica. Química Nova, 32: 2151-2158. DOI: https://doi.org/10.1590/s010040422009000800029.

Brasil, 2008. Ministério da Agricultura, Pecuária eAbastecimento. Instrução Normativa $\mathrm{N}^{\circ} 41$, de $1^{\circ}$ de julho de 2008. Altera os Anexos I e II da Instrução Normativa $\mathrm{n}^{0} 52$, de 20 de novembro de 2007, que passa a vigorar na forma dos Anexos à presente Instrução Normativa. Diário Oficial [da] República Federativa do Brasil, Brasília, DF, n. 125, 02 jul. 2008. Seção 1, p. 8.

Brasil, 2018. Ministério da Agricultura, Pecuária e Abastecimento. Instrução Normativa $\mathrm{N}^{\circ} 38$, de $1^{\circ}$ de outubro de 2018. Diário Oficial [da] União, Brasília, DF, 2 out. 2018, Seção 1, p. 14.

Calgaro, M \& MB Braga, 2012. A cultura da acerola. 3 ed. rev. e ampl. Brasília, Embrapa. (Coleção Plantar, 69), 144 p.

Clark, WE, 1987. Revision of the unipustulatus group of the weevil genus Anthonomus Germar (Coleoptera: Curculionidae). The Coleopterists Bulletin, 41: 73-88.

Freitas, CAS., GA Maia, JMC Costa, RW Figueiredo \& PHM Sousa, 2006. Acerola: produção, composição, aspectos nutricionais e produtos. Revista Brasileira de Agrociência, 12: 395-400.

IBGE, 2017. Censo Agro 2017 - Acerola Brasil. [Rio de Janeiro, 2017]. Available on: <https://censos.ibge.gov. br/agro/2017/templates/censo agro/resultadosagro/ agricultura.html?localidade $=0 \&$ tema $=76215>$. [Accessed in: 17.viii.2018].
Jarquín-López, R, L Martínez-Martínez, JA SánchezGarcía \& JI Figueroa, 2011. Parasitoides asociados a Anthonomus sisyphus Clark (Coleoptera: Curculionidae) en frutos de nanche rojo (Malpighia mexicana) en Oaxaca, México. Southwestern Entomologist, 36: 351-361. DOI: https://doi.org/10.3958/059.036.0312.

Lemos, LN, JDB Pereira, RA Silva \& AL Marsaro Júnior, 2011. Vulnerabilidade da faixa de fronteira do Brasil à introdução de espécies invasoras exóticas, pp. 121-144. In: Porto, JLR \& ED Sotta (Orgs.) Reformatações fronteiriças no Platô das Guianas: (re)territorialidades de cooperações em construções. Rio de Janeiro, Publit, 224 p.

Marsaro Júnior, AL, PRV. Pereira, GH Rosado-Neto \& EGF Morais, 2017. First record of acerola weevil, Anthonomus tomentosus (Faust, 1894) (Coleoptera: Curculionidae), in Brazil. Brazilian Journal of Biology, 77: 803-808. DOI: https://doi.org/10.1590/1519-6984.01216.

Morais, EGF, CR Jesus-Barros, R Adaime, AL Lima \& D Navia, 2016. Pragas de expressão quarentenária na Amazônia, pp. 520-559. In: Silva, NM, R Adaime \& RA Zucchi (Eds.). Pragas Agrícolas e Florestais na Amazônia. Brasília: Embrapa, 608 p.

Nogueira, RJMC, JAPV Moraes, HA Burity \& JF Silva Junior, 2002. Efeito do estádio de maturação dos frutos nas características físico-químicas de acerola. Pesquisa Agropecuária Brasileira, 37: 463-470. DOI: https://doi.org/10.1590/s0100-204X2002000400006.

\section{Suggestion citation:}

Adaime, R, RP Almeida, JF Pereira, AL Marsaro Júnior \& GH Rosado-Neto, 2019. Primeiro registro de Anthonomus tomentosus (Faust) (Coleoptera: Curculionidae) na Amazônia Oriental. EntomoBrasilis, 12 (2): 84-87.

Available on: doi:10.12741/ebrasilis.v12i2.814

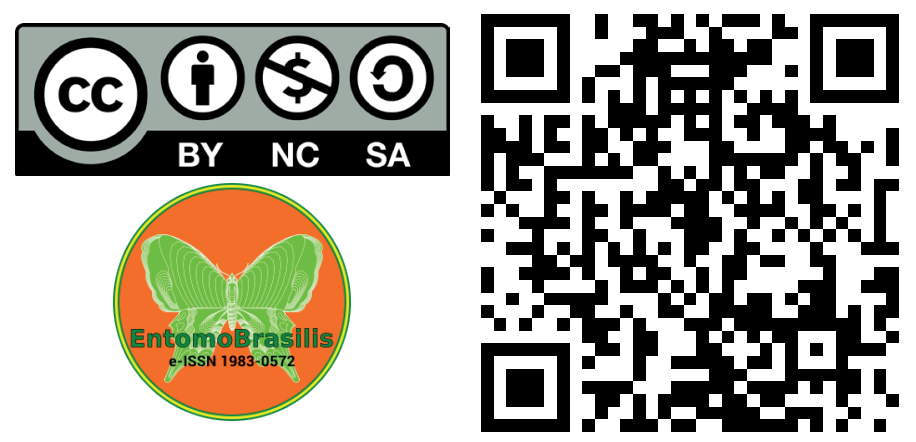

